\title{
A New Kalman Filtering Method to Resist Motion Model Error In Dynamic Positioning
}

\author{
Chengsong Zhou ${ }^{\mathrm{a}}$, Jing Peng, Wenxiang Liu, Feixue Wang \\ Electronic Science and Engineering School, National University of Defense Technology. Changsha \\ 410073,China \\ awhunudter@whu.edu.cn
}

Keywords: motion model error, adaptive robust kalman filtering, predictive state errors, standardized residuals.

\begin{abstract}
Against the disadvantage of traditional kalman filtering which is susceptible to the influence produced by the motion model error, this paper proposes a new kalman filtering method which can resist the motion model error. This new method uses the standardized residuals of the predictive position errors to choose the appropriate least-square principle between two kinds of recursive least-square principles adaptively, so as to achieve the purpose of the robust filtering. The experimental results show that, this new method can effectively weaken the effect of motion model error and track the moving vehicle's trajectory accurately in complex movement environment.
\end{abstract}

\section{Introduction}

If the kalman filtering is applied to dynamic navigation positioning, a more reasonable and accurate motion model come first ${ }^{[1]}$. Domestic and foreign scholars studied the motion model of the motor vehicle and put forward the differential polynomial model, uniform model, the acceleration model, time correlation model, the "current" statistical model, etc ${ }^{[2]}$. But each kind of the motion model is only suitable for the corresponding motor case. When the motion model is not suitable, it could lead to poor filtering effect, even lead to the divergence ${ }^{[3]}$.

The predictive state errors of the kalman filtering could reflect the accuracy of the motion model to a certain degree ${ }^{[4]}$. In order to restrain the motion model error, this paper use the standardized residuals of the vehicle's predictive position errors produced by kalman filtering to choose the appropriate least-square principle between two kinds of recursive least-square principles adaptively and so as to achieve the purpose of the robust filtering.

\section{Kalman Filtering}

\subsection{The Kalman Filtering Algorithm}

Kalman filtering is based on state equation and observation equation ${ }^{[5]}$, they are

$$
\begin{aligned}
& X_{k}=\phi_{k, k-1} X_{k-1}+W_{k} \\
& L_{k}=A_{k} X_{k}+e_{k}
\end{aligned}
$$

In (2.1), $X_{k}$ is The state vector at epoch $t_{k} ; \phi_{k, k-1}$ is the state transition matrix; $W_{k}$ is the state model noise; In (2.2), $L_{k}$ is the observation vector at epoch $t_{k} ; A_{k}$ is the observation designed matrix; $e_{k}$ is the observation noise.

Performing the kalman filter based on (2.1) and (2.2), then get the state estimation vector $\hat{X}_{k}$, its concrete steps are from (2.3) to (2.9).

$$
\begin{aligned}
& \bar{X}_{k}=\phi_{k, k-1} \hat{X}_{k-1} \\
& \xi_{\bar{X}_{k}}=\phi_{k, k-1} \xi_{\hat{X}_{k-1}} \phi_{k, k-1}^{T}+\xi_{W_{k}} \\
& \bar{V}_{k}=A_{k} \bar{X}_{k}-L_{k} \\
& \xi_{\bar{V}_{k}}=A_{k} \xi_{\bar{X}_{k}} A_{k}^{T}+\xi_{k}
\end{aligned}
$$




$$
\begin{aligned}
& K_{k}=\phi_{k, k-1} A_{k} \xi_{\bar{v}_{k}}^{-1} \\
& \hat{X}_{k}=\bar{X}_{k}-K_{k} \bar{V}_{k} \\
& \xi_{\hat{X}_{k}}=\xi_{\bar{X}_{k}}-\xi_{\bar{X}_{k}} A_{k}^{T}\left(A_{k} \xi_{\bar{X}_{k}} A_{k}^{T}+\xi_{k}\right)^{-1} A_{k} \xi_{\bar{X}_{k}}
\end{aligned}
$$

In (2.3), $\bar{X}_{k}$ is the predicted state vector, $\phi_{k, k-1}$ denotes the state transition matrix from epoch $t_{k-1}$ to epoch $t_{k}, \hat{X}_{k-1}$ is the state estimation vector at epoch $t_{k-1}$. In (2.4), $\xi_{\bar{X}_{k}}$ is the state predicted covariance at epoch $t_{k}, \xi_{\hat{x}_{k-1}}$ is the state estimation covariance matrix at epoch $t_{k-1}, \xi_{W_{k}}$ is the covariance matrix of motion model noise. In (2.5), $\bar{V}_{k}$ is the innovation vector $A_{k}$ is the observation matrix, $L_{k}$ is the observation vector. In (2.6), $\xi_{\bar{V}_{k}}$ is the covariance matrix of innovation vector, $\xi_{k}$ is the covariance matrix of observation vector. In (2.7), $K_{k}$ is the gain matrix. In (2.8), $\hat{X}_{k}$ is the state estimation vector at epoch $t_{k}$. In (2.9), $\xi_{\hat{X}_{k}}$ is the state estimation covariance matrix at epoch $t_{k}$.

\subsection{Solving Principles of Kalman Filtering}

It can get the different filtering results based on different solving principles ${ }^{[6]}$. This paper uses two kinds of least-square criterion for filtering, one considers the motion model errors and the observation model errors as a whole, then establishes the least-square principle as (2.10).

$$
\Omega_{1}(k)=V_{k}^{T} \xi_{k}^{-1} V_{k}+V_{\bar{x}_{k}}^{T} \xi_{\bar{x}_{k}}^{-1} V_{\bar{x}_{k}}=\min \quad(2.10)
$$

In (2.10), $V_{k}$ is the residual vector at epoch $t_{k}$, such as (2.11). $V_{\bar{x}_{k}}$ is the predictive state error at epoch $t_{k}$, such as (2.12).

$$
\begin{aligned}
& V_{k}=A_{k} \hat{X}_{k}-L_{k} \\
& V_{\bar{x}_{k}}=\bar{X}_{k}-\hat{X}_{k}
\end{aligned}
$$

The other one is the least-square principle which could resist the motion model error, such as (2.13).

$$
\Omega_{2}(k)=V_{k}^{T} \xi_{k}^{-1} V_{k}=\min
$$

\subsection{Choosing From The Two Least-square Principles}

To solve the problem how to choose one from the two least-square principles adaptively, such as (2.10) and (2.13), this paper uses the standardized residuals of the predictive position errors to choose the appropriate least-square principle between two kinds of recursive least-square principles adaptively, so as to achieve the purpose of the robust filtering ${ }^{[4]}$.

The position values of the predictive state vectors at each epoch which calculated by (2.3) is $\left[\bar{X}_{1}, \bar{X}_{2}, \mathrm{~L}, \bar{X}_{k}\right]^{T}$, and $\bar{X}_{k}=\left[\bar{X}_{k}, \bar{y}_{k}, \bar{z}_{k}\right]^{T}$; the position values of the state estimation vectors at each epoch which calculated by $(2.8)$ is $\left[\hat{X}_{1}, \hat{X}_{2}, \mathrm{~L}, \hat{X}_{k}\right]^{T}$, and $\hat{X}_{k}=\left[\hat{X}_{k}, \hat{y}_{k}, \hat{z}_{k}\right]^{T}$. The predictive position errors at epoch $t_{k}$ are calculated by (2.14). Thus it can obtain the predictive position errors at each epoch, such as $\left[V_{\bar{X}_{1}}, V_{\bar{X}_{2}}, \mathrm{~L}, V_{\bar{X}_{k}}\right]$.

$$
V_{\bar{X}_{k}}=\bar{X}_{k}-\hat{X}_{k}=\left[V_{\bar{x}_{k}}, V_{\bar{y}_{k}}, V_{\bar{z}_{k}}\right]^{T}
$$

The standardized residuals at each epoch could be calculated by (2.15) based on the predictive position errors (except the first epoch).

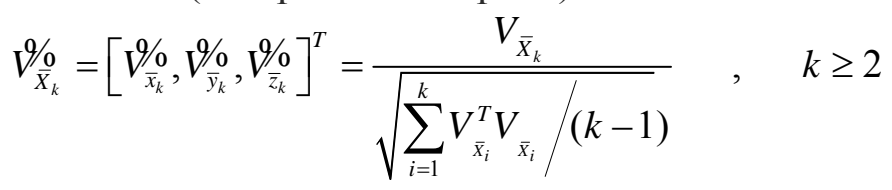

Then choose the least-square principle through the standardized residuals at each epoch, and the way for choosing as follow.

The standardized residuals of the predictive position errors are not large at epoch $t_{k}$ when the values of $V \%, V \%, V \%$ are less than 1 , that suggests the motion model of the kalman filtering is 
suitable with the actual motor case, thus the least-square principle which considers the motion model errors and the observation model errors as a whole should be chosen, such as (2.10). If the values of $V \frac{V}{\bar{X}_{k}}, V_{V_{k}}, V_{\bar{z}_{k}}$ are larger than 1 , the least-square principle which resists the motion model errors should be chosen, such as (2.13). In addition, the least-square principle which resists the motion model errors should be chosen when $t_{k}$ is 1 .

\section{The Measured Data Experimental}

\subsection{The Observation Equation}

Driving a vehicle that equipped with a GPS receiver whose sampling interval is 1 second in urban areas for $1.5 \mathrm{~h}$. With the extended kalman filtering method ${ }^{[7]}$, using the pseudo-ranges for measuring the vehicle's position and doppler frequencies for measuring the vehicle's velocity ${ }^{[8]}$.Setting the state vector of kalman filtering like $\left[x, \dot{x}, \ddot{x}, y, \dot{y}, \ddot{y}, z, \dot{z}, \ddot{z}, b_{u}, \dot{b}_{u}\right]^{T}$, and the state vector is 11 dimensions which is composed of GPS receiver's three-dimensional positions, velocities and accelerations in ECEF coordinate system, the receiver clock error and clock rate. Then make the pseudo- range observation equation expand at the receiver's approximate position which is $\left(\hat{x}, \hat{y}, \hat{z}, \hat{b}_{u}\right)$ by using Taylor series method ${ }^{[9]}$, thus get (3.1).

$$
\rho_{j u}=R_{j}+\hat{b}_{u}+v_{j}-\frac{x_{j}-\hat{x}_{u}}{R_{j}} \Delta x_{u}-\frac{y_{j}-\hat{y}_{u}}{R_{j}} \Delta y_{u}-\frac{z_{j}-\hat{z}_{u}}{R_{j}} \Delta z_{u}+\Delta b_{u}
$$

In (3.1), $\rho_{j u}$ is the corrected pseudo-range from satellite $j$ to the receiver $u, R_{j}$ is the distance from satellite $j$ to the receiver's approximate position, $v_{j}$ is the corrected pseudo-range error, $\left(\Delta x_{u}, \Delta y_{u}, \Delta z_{u}\right)$ is the receiver 's position correction; $\Delta b_{u}$ is the receive clock error correction.

To measure the vehicle's velocity, take the derivative of (3.1) with respect to the time, then get (3.2).

$\dot{\rho}_{j u}=\dot{b}_{u}+\dot{v}_{j}+\frac{x_{j}-\hat{x}_{u}}{R_{j}}\left(\dot{x}_{j}-\dot{x}_{u}\right)+\frac{y_{j}-\hat{y}_{u}}{R_{j}}\left(\dot{y}_{j}-\dot{y}_{u}\right)+\frac{z_{j}-\hat{z}_{u}}{R_{j}}\left(\dot{z}_{j}-\dot{z}_{u}\right)$

According to (3.1) and (3.2), the observed equation becomes (3.3).

$$
V=A X-L
$$

In (3.3), $A$ is the observation matrix, $L$ is the constant vector, $V$ is the residual vector which can be ignored.

\subsection{State Equation}

This paper uses acceleration model as motion model of the kalman filtering, and the state vector is $\left[x, \dot{x}, \ddot{x}, y, \dot{y}, \ddot{y}, z, \dot{z}, \ddot{z}, b_{u}, \dot{b}_{u}\right]^{T}$, thus the state equation is

$$
\begin{gathered}
X_{k}=\left[\begin{array}{cccc}
\phi_{x_{k, k-1}} & 0 & 0 & 0 \\
0 & \phi_{y_{k, k-1}} & 0 & 0 \\
0 & 0 & \phi_{z_{k, k-1}} & 0 \\
0 & 0 & 0 & \phi_{z_{k, k-1}}
\end{array}\right] X_{k-1}+W_{k} \\
\phi_{x_{k, k-1}}=\phi_{y_{k, k-1}}=\phi_{z_{k, k-1}}=\left[\begin{array}{ccc}
1 & \Delta t & \Delta t^{2} / 2 \\
0 & 1 & \Delta t \\
0 & 0 & 1
\end{array}\right] \\
\phi_{c_{k, k-1}}=\left[\begin{array}{cc}
1 & \Delta t \\
0 & 1
\end{array}\right]
\end{gathered}
$$




\subsection{The Result of The Experiment}

Use the positioning trajectory which is calculated through the high-precision weighted least-square pseudo-range positioning method as the receiver's reference- trajectory. The high-precision weighted least-square pseudo-range positioning is based on the real-time satellite altitude angle, and adjusts the weight of the satellite's data for calculating the receiver's position, reduces the problem satellites' weights and improves healthy satellites' weights, finally improves the accuracy of positioning ${ }^{[10]}$.

Take the receiver's observations to be filtered through the traditional kalman filtering and the new kalman filtering which can resist the motion model error. Then make the two filtering results compare with the reference-trajectory, the comparison results of $x y$ directions are shown in figure 1, figure 2, the positioning errors of $x y$ directions are shown in figure 3, figure 4 .

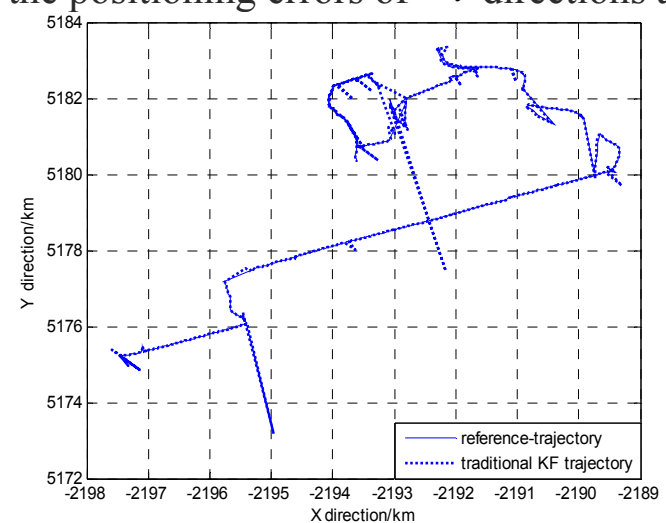

Fig 1 Trajectory of traditional KF
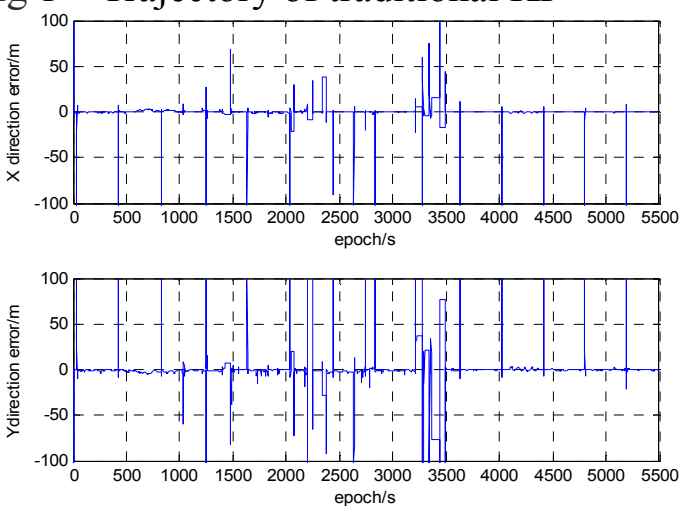

Fig $3{ }^{x y}$ direction error of traditional KF

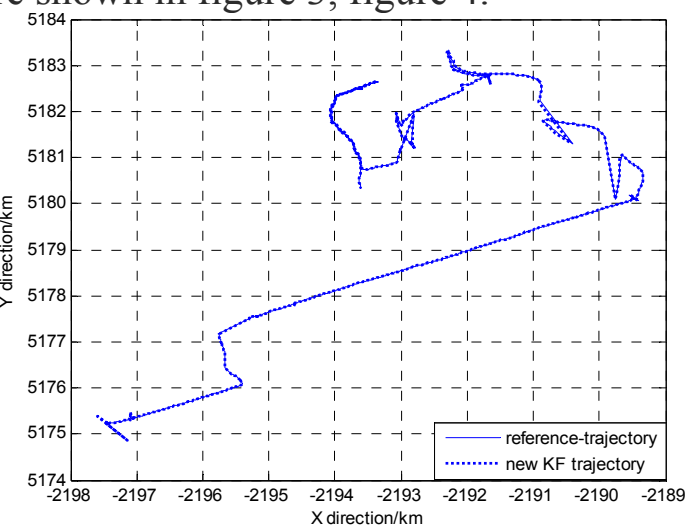

Fig 1 Trajectory of new KF
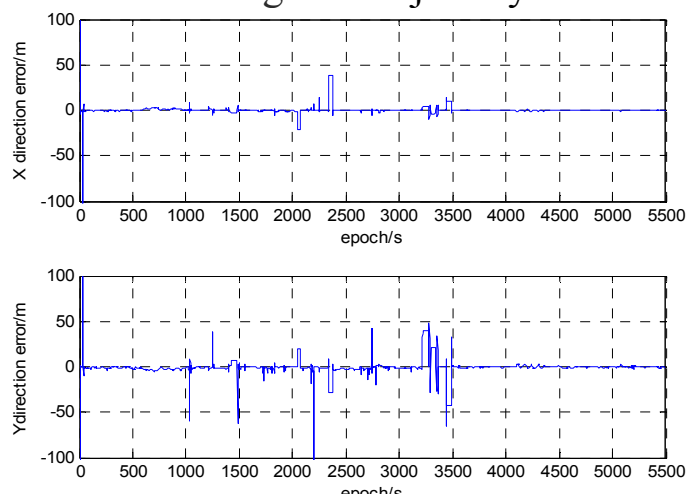

Fig $4 x y$ direction error of new KF

Table 1 The average of positioning errors

\begin{tabular}{cccc}
\hline & $x$ & $y$ & $z$ \\
\hline Traditional KF & 0.558 & -3.556 & -3.471 \\
New KF & -0.230 & 0.617 & 0.236 \\
\hline & Table 2 & The variance of positioning errors \\
\hline \multicolumn{4}{c}{$x$} \\
\hline Traditional KF & 402.411 & $y$ & $z$ \\
New KF & 34.959 & 7678.278 & 3869.949 \\
\hline
\end{tabular}

\section{Experimental Results Analysis}

Analysis figure 1 to figure 4, the positioning accuracy of the new kalman filtering method resisting motion model errors is higher than the traditional kalman filtering method's, and the new method can resist motion model errors to a great extent, then make an accurate navigation and positioning. But the trajectory of the traditional kalman filtering method has so many divergences that its positioning errors are larger, it cannot be used in dynamic positioning if the motion model is larger different from the vehicle's actual motor case.

Analysis table 1 to table 2, the positioning errors' average of the new kalman filtering method 
resisting motion model errors is dm level, and positioning errors' variance is smaller. But the positioning errors' mean value of the tradition kalman filtering method is more than $3 \mathrm{~m}$, and its variance is larger.

As shown in figure 1 and figure 2, the vehicle trajectory is very complex, real motion model contains uniform motion, uniformly accelerated motion, variable accelerated motion, turning motion, sudden stopping and starting motion, etc, so it is difficult to estimate accurately. And this experiment uses uniformly accelerated motion as the motion model, thus it must cause large motion model errors in the complex real motion scene that the traditional kalman filtering cannot make an accurate positioning, however the new kalman filtering method proposed in this paper can make an accurate positioning.

As shown in figure 3 and figure 4, there are many diverging results by using the traditional kalman filtering method and some positioning errors are larger than $100 \mathrm{~m}$. Though there is no diverging result by using the new kalman filtering method, positioning error of $50 \mathrm{~m}$ still appears and even one is larger than $100 \mathrm{~m}$. That is because the results of the kalman filtering are affected by motion model errors and observation model errors at the same time ${ }^{[11]}$. And the kalman filtering method this paper proposed can resist the motion model errors effectively that there is no diverging result, but large positioning error appears if the observation model error is large. And the experiment is conducted in the urban area so that the observation model error is large when the multipath effect that caused by the obstacles such as tall buildings is serious. Therefore the observation model error is large at some epochs.

\section{Summary}

In the experiment of this paper, the vehicle moves in the complex real scene so that the motion model of the kalman filtering method may be not suitable for the real motion model. In this case some positioning errors of the traditional kalman filtering method are larger than $100 \mathrm{~m}$, even some diverging results appear. However only a little bit positioning errors of the new kalman filtering method reach $50 \mathrm{~m}$ level at the same case and the $50 \mathrm{~m}$ positioning errors are due to the observation model error. But overall, the new kalman filtering method can track the vehicle's real trajectory accurately, and it can make an accurate navigation and positioning while the satellite observation condition is fine.

\section{References}

[1] Yuanxi Yang. Adaptive navigation and kinematic positioning. Publishing House of Surveying and Mapping ,2006,p. 69-70.

[2] Liang Chen. Research on key technology of maneuvering target tracking.(Doctor Thesis, Harbin engineering university,China 2012).p. 5-8

[3] Fumei Wu,Yuanxi Yang .A New Two-Step Adaptive Robust Kalman Filtering in GPS/INS Integrated Navigation System. Acta Geodaetica et Cartographica Sinic- a. Vol. 39(2010) No.5, p. 522-523.

[4] Fumei Wu,Yuanxi Yang,Xianqiang Cui.Application of Adaptive Factor Based on Partial State Discrepancy in Tight Coupled GPS /INS Integration. Geomatics and Information Science of Wuhan University. Vol. 35(2010) No.2, p. 156-157.

[5] Yuangxi Yang,Xia Ren,Yan Xu.Main progress of adaptively robust filter with ap- plications in navigation.Journal of navigation and positioning. Vol. 1(2013) No.1, p. 9-15.

[6] Pengfei Luo. Fundamentals of statistical signal processing . Publishing House of Electronics Industry ,2014,p. 289-311.

[7] Gang Xie. Principles of GPS and Receiver Design. Publishing House of Electron- ics Industry ,2009,p. 152-153. 
[8] Aiyu Yuan,Honglei Qin.Applications of UKF in GPS/INS Pseudorange and Pse- udorange Rate Integrated Navigation. Radar Science and Technology, Vol. 7(2009) No.1, p. 59-64.

[9] Haibo He,Yuanxi Yang,Zhongmiao Sun .Mathematic Model and Error Analyses for Velocity Determination Using GPS Doppler Measurements. Journal of Insti- tute of Surveying and Mapping, Vol. 20(2003) No.2, p. 79-82.

[10]Chunhua Li ,Chenglin Cai ,Yugao Liang.A high-precision weighted least squares algorithm for BDS pseudo-range single point positioning.Science of Surveying and Mapping, Vol.40(2015) No.9, p. 33-38.

[11]Yuanxi Yang, Weiguang Gao.Comparison of Two Fading Filters and Adaptively Robust Filter.Geo-spatial Information Science, Vol. 10(2007) No.3, p. 200-203. 